\title{
Neoaortic root dilatation with saccular aneurysm formation after the arterial switch operation for Taussig-Bing anomaly
}

\author{
Yoshitaka Hayashi, MD, PhD, ${ }^{a}$ Andrew D. Cochrane, MBBS, FRACS, ${ }^{a}$ \\ Samuel Menahem, MD, BS, FRACP, FACC, FCSANZ, ${ }^{\mathrm{b}}$ and \\ Julian A. Smith, MS, FRACS, a,c Clayton, Victoria, Australia
}

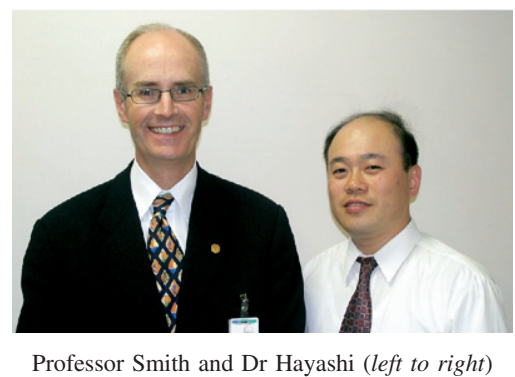

$\mathrm{T}$

he arterial switch operation (ASO) has become the preferred procedure for repair of d-loop transposition of the great arteries, and this anatomic correction has been applied to the treatment of right ventricular dysfunction after atrial switch procedures, as well as primary curative repair in newborns. On the other hand, this operation involves radical manipulation of the base of the great arteries. Both great arteries are transected and reanastomosed above the sinuses of Valsalva, and the coronary arteries are translocated. The native pulmonary valve becomes the systemic outflow valve, and the anatomic pulmonary root is subjected to systemic blood pressure. Neoaortic root dilatation, which is one of the postoperative complications after ASO, is thought to occur as a result of these atypical hemodynamic and anatomic features. ${ }^{1}$

Neoaortic root dilatation might be recognized during late followup, ${ }^{2,3}$ and some patients might require a neoaortic root or valve operation at some stage after an ASO. ${ }^{3,4}$ Careful assessment of the individual anatomic situation is essential before surgical repair because coronary artery distribution and the positional relationship of the great arteries affect the conduct of the operative procedure. We describe a case of neoaortic root dilatation accompanied by a huge saccular aneurysm, which was detected during pregnancy. The patient had undergone an ASO for Taussig-Bing anomaly (double-outlet right ventricle with subpulmonary ventricular septal defect) 14 years previously. We also discuss some of the important issues regarding the assessment of neoaortic root dilatation after an ASO.

\section{Clinical Summary}

The patient, a 26-year-old woman, was born with the TaussigBing anomaly accompanied by subpulmonary stenosis. She underwent a Senning operation at the age of 8 months as a radical repair

From the Cardiothoracic Surgery Unit ${ }^{\mathrm{a}}$ and the Department of Paediatric Cardiology, ${ }^{\mathrm{b}}$ Monash Medical Centre, Clayton, Victoria, Australia, and the Department of Surgery (MMC), ${ }^{\mathrm{c}}$ Monash University, Clayton, Victoria, Australia.

Received for publication Sept 12, 2006; accepted for publication Sept 19, 2006.

Address for reprints: Julian A. Smith, MS, FRACS, Cardiothoracic Surgery Unit, Monash Medical Centre, 246 Clayton Rd, Clayton, Victoria 3168, Australia (E-mail: julian.smith@med.monashi.edu.au).

J Thorac Cardiovasc Surg 2007;133:569-72

$0022-5223 / \$ 32.00$

Copyright () 2007 by The American Association for Thoracic Surgery

doi:10.1016/j.jtcvs.2006.09.047

after balloon atrial septostomy on her third day of life. At the age of 2.5 years, she subsequently had a resection of the subpulmonary fibrous membrane and muscle for moderate-to-severe left ventricular outflow tract obstruction. For the treatment of progressive right ventricular dysfunction, she underwent banding of the pulmonary artery, followed 9 months later by an ASO and a takedown of the Senning repair at the age of 12.5 years. Her coronary anatomy was type $\mathrm{E}$ of Yacoub's classification; the right coronary artery (RCA) and the left anterior descending branch took origin from the left ostium, and the circumflex branch alone took origin from the right ostium. A concomitant neoaortic valve replacement with a 21-mm SJM mechanical valve (St Jude Medical, Inc, St Paul, Minn) was required because of massive neoaortic regurgitation caused by an abnormal native pulmonary valve. The left anterior neoaortic sinus was reconstructed with the use of an autologous pericardial patch to prevent obstruction of the leftsided coronary artery. She did well for 14 years until late in her first pregnancy, when she was found to have an asymptomatic saccular aneurysm of the neoaortic root. Chest radiography showed an abnormal mediastinal shadow and mild cardiomegaly (cardiothoracic ratio, 54\%; Figure 1, A).

Her echocardiogram showed a mildly dilated left ventricle with mild eccentric left ventricular hypertrophy and normal left ventricular function. The mechanical valve at the neoaortic site appeared to be opening and closing normally with a moderate transvalvular gradient (peak gradient, $38 \mathrm{~mm} \mathrm{Hg}$; mean gradient, 24 $\mathrm{mm} \mathrm{Hg}$ ), and no significant regurgitation was detected. Marked dilatation of the neoaortic root with aneurysmal sinuses of Valsalva extending anteromedially and superiorly toward the aortic arch was observed. The maximum diameter was at least $70 \mathrm{~mm}$. Turbulent flow was noted within the aneurysm, and there was no evidence of a dissection flap. Right ventricular size and function were normal. Previous echocardiography done 18 months before had not revealed either critical neoaortic dilatation or saccular aneurysm formation.

Multisliced computed tomography (CT) and magnetic resonance imaging clearly delineated the neoaortic root and coronary arterial anatomy. Neoaortic root dilatation was shown with a lobulated saccular aneurysm in the left anterior sinus, measuring $64 \times 88 \times 50 \mathrm{~mm}$ and extending leftward in the chest (Figure 1, $B)$. There was no intimal flap, thrombus, or extra saccular fluid collection. The main pulmonary artery was located and displaced to the right anteriorly and marginally compressed by the aneurysm (Figure 2). The RCA originated anteromedially, about $3.1 \mathrm{~cm}$ above the aortic valve from the right anterior sinus. The left anterior descending branch arose (just distal) at the origin of the RCA. The circumflex branch originated posteromedially from the 


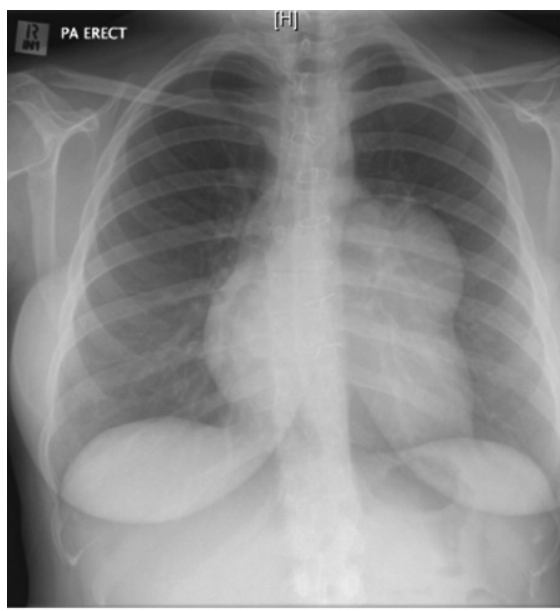

A
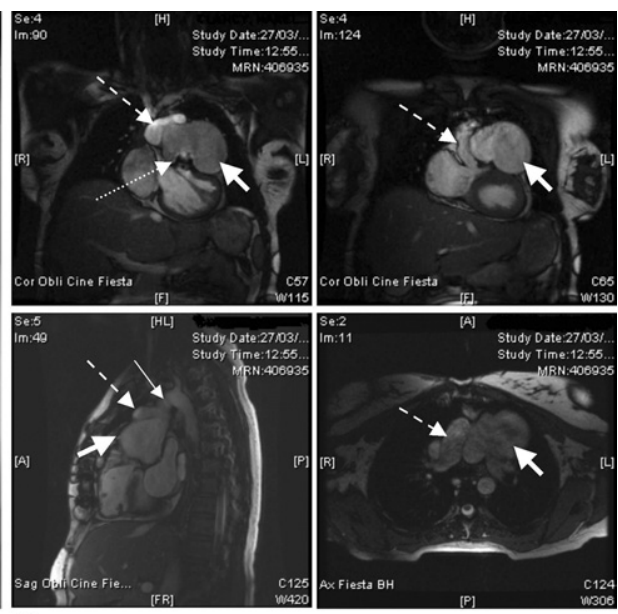

B

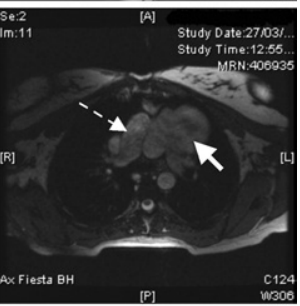

Figure 1. A, Chest radiograph showing an abnormal mediastinal shadow and mild cardiomegaly. B, Magnetic resonance imaging revealing neoaortic root dilatation with a $64 \times 88 \times 50 \mathrm{~mm}$ saccular aneurysm in the left anterior sinus extending leftward in the chest (arrows: bold solid line, neoaortic root aneurysm; solid line, distal ascending aorta; broken line, main and left pulmonary artery, dotted line, prosthetic valve). left posterior sinus (at the level of the aortic root), which was compressed by the huge aneurysm (Figure 2). Neither CT angiography nor coronary angiography revealed significant coronary artery narrowing.

On detection of this pathology, the patient was admitted to the hospital and started on atenolol. Her warfarin had been converted to twice-daily subcutaneous heparin at the start of her planned pregnancy. Several days after admission, she experienced a central abdominal pain, and investigation revealed a retroplacental hemorrhage. She underwent a caesarean section at 30 weeks with delivery of a healthy infant without congenital heart disease. Three weeks later, she underwent her fifth cardiac operation.
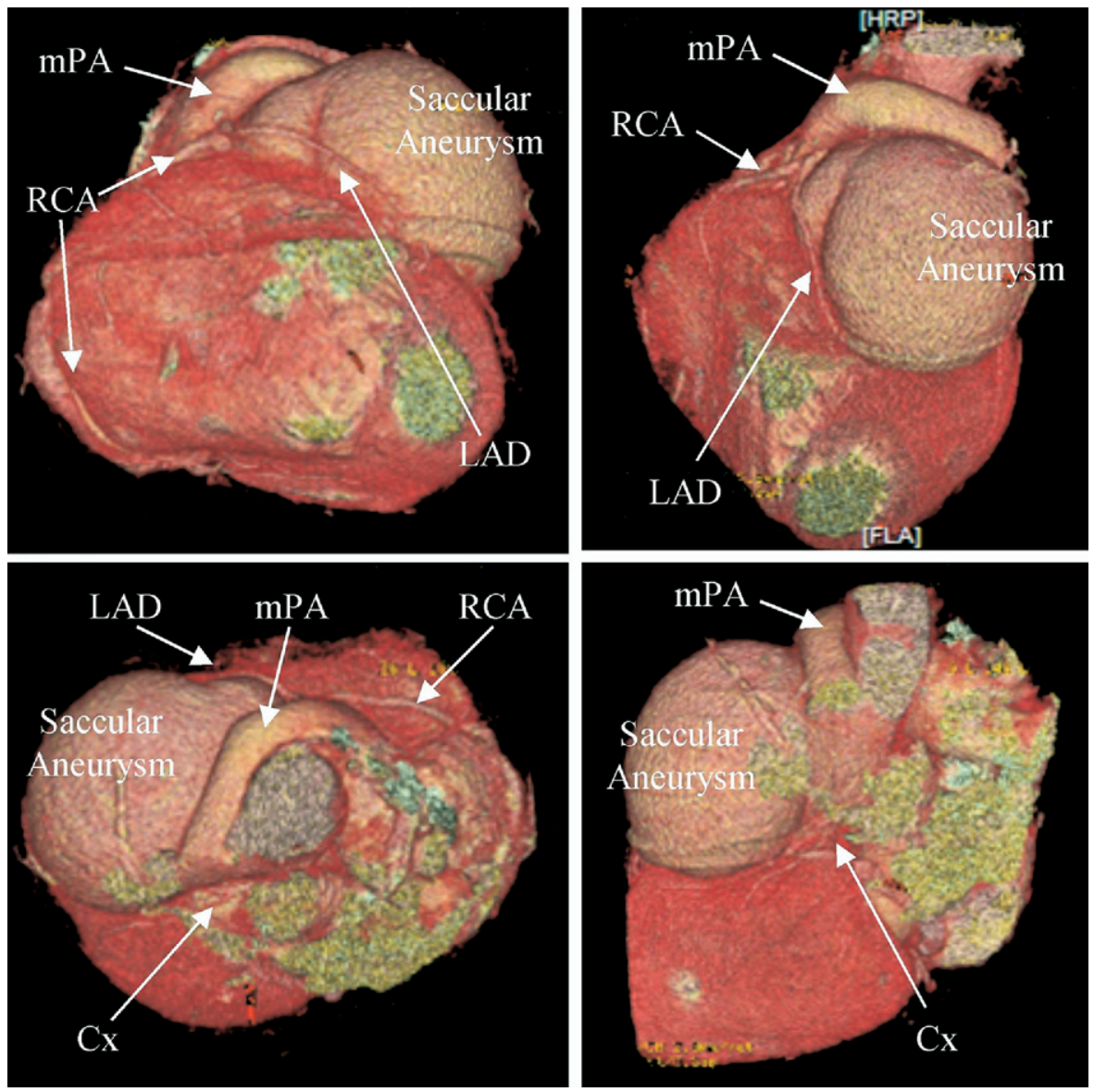

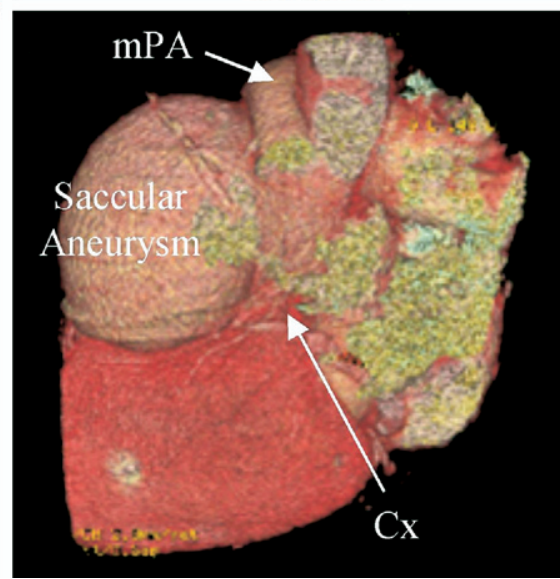

Figure 2. Multislice computed tomographic angiography showing the relationship between the great arteries and the coronary arteries. RCA, Right coronary artery; $L A D$, left anterior descending coronary artery; $C_{x}$, circumflex branch of the coronary artery; $m P A$, main pulmonary artery. 
The proximal ascending aorta and neoaortic root were grossly dilated, with significant effacement of the coronary ostia. The saccular aneurysm extended laterally into the left hemithorax. The distal ascending aorta was $3.0 \mathrm{~cm}$ in diameter and minimally diseased. After systemic heparinization, an arterial cannula was inserted through the left femoral artery, and a 2-stage venous cannula was inserted through the right atrium. Cardiopulmonary bypass was established, and cardioplegic arrest was rapidly achieved and maintained by means of the infusion of initial and intermittent boluses of antegrade and retrograde cold blood cardioplegia. The left pulmonary artery was divided to facilitate a sufficient exposure to the ascending aorta, and the ascending aorta was transected. The 21-mm SJM mechanical prosthesis was well seated, with minimal pannus formation. The wall of the aorta was extremely thin, and its aneurysmal change extended around the entire neoaortic annulus, which indicated that a redo aortic root replacement was necessary. The previously inserted mechanical prosthesis was removed with the debridement of the annulus, and a 21-mm aortic mechanical valved graft (ATS OPEN PIVOT; ATS Medical, Inc, Minneapolis, Minn) was placed in a supra-annular position. Subsequently, the coronary ostia were mobilized with ease and anastomosed to the conduit. A terminal warm blood cardioplegia was delivered after the completion of distal conduit anastomosis. Spontaneous cardiac rhythm was restored after release of the aortic crossclamp. The left pulmonary artery was reconstructed during reperfusion with a 21-mm interposition Dacron graft to reestablish the continuity without tension. The patient was weaned from cardiopulmonary bypass with the assistance of low-dose inotropes. The postoperative course was uneventful, and she was discharged from the hospital on the seventh postoperative day. She remains well 6 months later, with an excellent surgical result seen on echocardiography.

\section{Discussion}

Neoaortic root dilatation has been reported as one of the postoperative complications after an ASO. There is usually a rapid dilatation of the neoaortic root in the early postoperative period, and the root dimensions tend to stabilize without significant progression over time. ${ }^{2,3}$ However, progressive development of neoaortic root dilatation can be associated with significant complications, and thus, close postoperative monitoring of the neoaortic root is essential.

Many factors can influence the development of neoaortic root dilatation after ASO. Murakami and colleagues ${ }^{1}$ demonstrated the impaired distensibility of neoaorta after ASO in 36 patients and suggested 3 possible mechanism of neoaortic root dilatation: (1) "surgical manipulation such as the suture of coronary buttons," (2) "characteristics of the native pulmonary artery in a high-pressure position," and (3) "damage to the vasa vasorum."1 Schwartz and associates ${ }^{3}$ identified the most significant risk factor as previous pulmonary artery banding in 335 patients who underwent ASO for primary repair of d-loop transposition of the great arteries or Taussig-Bing anomaly. In a retrospective longitudinal study of 144 patients, Hutter and coworkers ${ }^{2}$ observed the growth of the neoaortic valve and neoaortic root by means of echocardiography and evaluated the influence of associated cardiac malformations, such as ventricular septal defect, Taussig-Bing anomaly, and coarctation of the aorta. Furthermore, false aneurysm for- mation was common in patients who underwent cardiac operations with the use of prosthetic materials, such as an artificial valve or a pericardial patch. ${ }^{5-7}$ In consideration of the risk factors mentioned above, our patient was considered to be at a higher risk.

Saccular aneurysms are mostly observed in patients who underwent a patch aortoplasty for repair of coarctation of the aorta., This aneurysm formation is considered a rare life-threatening complication after aortic surgery as a result of disruption of at least 1 layer of the vascular wall. McGiffin and associates ${ }^{10}$ described the aortic wall stress profile after the coarctation repair with a patch aortoplasty. In their mathematic models the authors suggested that excessive aortic wall stress caused by patch geometry can be responsible for aneurysm formation. Our patient had a pericardial patch for reconstruction of the left anterior coronary sinus to prevent compression of the left-sided coronary artery, and this might have lead to saccular aneurysm formation accompanied by neoaortic root dilatation.

Despite regular review and echocardiographic evaluation since her previous operation, the development of neoaortic root dilatation had not been observed, and her current pathology accompanied by saccular aneurysm was only detected during her first pregnancy when she was referred back to her cardiologist. Generally, cardiac output and stroke volume increase up to $30 \%$ to $50 \%$ by 16 weeks' gestation, and the peak level is sustained until 36 weeks' gestation. ${ }^{11,12}$ Therefore it might be postulated that acute development of her neoaortic pathology occurred during her pregnancy period. Studies have suggested that pregnancy might result in aortic root dilatation in patients with Marfan syndrome. ${ }^{13,14}$ There are no data on the influence of the pregnant state on congenitally abnormal hearts.

Preoperative anatomic evaluations are essential to clearly delineate the neoaortic root pathology and to plan surgical repair. The position of the great arteries and the coronary arterial pattern are critical pieces of information. Multidetection CT is particularly helpful, and its value in planning repeat cardiac operations has been demonstrated. ${ }^{15,16}$ Contrast-enhanced 3-dimensional images clearly visualized the anatomic relationship between the pathological neoaortic root and the coronary arteries in this case.

In summary, we have described a patient with neoaortic root dilatation accompanied by a huge saccular aneurysm formation after ASO, which was detected during her first pregnancy. Although neoaortic root dilatation is a rare complication after an ASO, careful follow-up of the neoaortic root and evaluation of risk of such dilatation are essential.

\section{References}

1. Murakami T, Nakazawa M, Momma K, Imai Y. Impaired distensibility of neoaorta after arterial switch procedure. Ann Thorac Surg. 2000; 70:1907-10.

2. Hutter PA, Thomeer BJ, Jansen P, Hitchcock JF, Faber JA, Meijboom EJ, et al. Fate of the aortic root after arterial switch operation. Eur J Cardiothorac Surg. 2001;20:82-8.

3. Schwartz ML, Gauvreau K, del Nido P, Mayer JE, Colan SD. Longterm predictors of aortic root dilatation and aortic regurgitation after arterial switch operation. Circulation. 2004;110(suppl II):II128-32.

4. Losay J, Touchot A, Serraf A, Litvinova A, Lambert V, Piot JD, et al. Late outcome after arterial switch operation for transposition of the great arteries. Circulation. 2001;104(suppl I):I121-6. 
5. Mulder EJ, van Bockel JH, Maas J, van den Akker PJ, Hermans J. Morbidity and mortality of reconstructive surgery of noninfected false aneurysm detected long after aortic prosthetic reconstruction. Arch Surg. 1998;133:45-9.

6. Mohammadi S, Bonnet N, Leprince P, Kolsi M, Rama A, Pavie A, et al. Reoperation for false aneurysm of the ascending aorta after its prosthetic replacement: surgical strategy. Ann Thorac Surg. 2005; 79:147-52.

7. Villavicencio MA, Orszulak TA, Sundt TM III, Daly RC, Dearani JA, McGregor CG, et al. Thoracic aorta false aneurysm: what surgical strategy should be recommended? Ann Thorac Surg. 2006;82:81-9.

8. Mesana TG, Caus T, Gaubert J, Collart F, Ayari R, Bartoli J, et al. Late complications after prosthetic replacement of the ascending aorta: what did we learn from routine magnetic resonance imaging followup? Eur J Cardiothorac Surg. 2000;18:313-20.

9. Roth M, Lemke P, Schönburg M, Klövekorn W-P, Bauer EP. Aneurysm formation after patch aortoplasty repair (Vossschulte): reoperation in adults with and without hypothermic circulatory arrest. Ann Thorac Surg. 2002;74:2047-50.
10. McGiffin DC, McGiffin PB, Galbraith AJ, Cross RB. Aortic wall stress profile after repair of coarctation of the aorta: is it related to subsequent true aneurysm formation? J Thorac Cardiovasc Surg. 1992;104:924-31.

11. Thorne SA. Pregnancy in heart disease. Heart. 2004;90:450-6.

12. Abbas AE, Lester SJ, Connolly H. Pregnancy and the cardiovascular system. Int J Cardiol. 2005;98:179-89.

13. Elkayam U, Ostrzega E, Shotan A, Hehra A. Cardiovascular problems in pregnant women with the Marfan syndrome. Ann Intern Med. 1995;123:117-22.

14. Meijboom LJ, Vos FE, Timmermans J, Boers GH, Zwinderman AH, Mulder BJ. Pregnancy and aortic root growth in the Marfan syndrome: a prospective study. Eur Heart J. 2005;26:914-20.

15. Gilkeson RC, Markowitz AH, Ciancibello L. Multisection CT evaluation of the reoperative cardiac surgery patient. Radiographics. 2003 23(suppl):S3-17.

16. Gasparovic H, Rybicki FJ, Millstine J, Unic D, Byrne JG, Yucel K, et al. Three dimensional computed tomographic imaging in planning the surgical approach for redo cardiac surgery after coronary revascularization. Eur J Cardiothorac Surg. 2005;28:244-9.

\title{
Congenital mitral stenosis: A rare presentation and novel approach to management
}

\author{
Rachel Pase Sosland, MD, James L. Vacek, MD, and Michael E. Gorton, MD, Kansas City, Kan
}

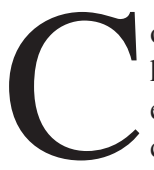

ongenital mitral stenosis is an uncommon congenital heart defect, typically presenting with symptoms in early childhood. ${ }^{1,2}$ This condition remains a surgical challenge, with a trend toward early single-stage complete repair. ${ }^{3}$ We present the case of a 15 -year-old patient given a diagnosis of severe mitral stenosis and pulmonary hypertension. The patient underwent successful complex mitral valve repair with mitral valve ring annuloplasty and neochordae placement and achieved dramatic improvement in symptoms and quality of life.

\section{Clinical Summary}

A 15-year-old boy with a history of asthma was transferred to our hospital with apparent status asthmaticus. He was initially treated for a 5-lobe pneumonia with intravenous antibiotic therapy. He was noted to have a loud holodiastolic murmur, best heard at the left lower sternal border. An echocardiogram was obtained that showed severe mitral stenosis (pressure half-time, $284 \mathrm{~cm} / \mathrm{s}$; mitral valve area, 0.8 $\mathrm{cm}^{2}$ ), significant tricuspid regurgitation, and an estimated systolic right ventricular pressure in excess of $100 \mathrm{~mm} \mathrm{Hg}$. His congestive heart failure responded favorably to diuresis; however, his shortness of breath continued, and he had difficulty attending school.

From the University of Kansas Hospital, Kansas City, Kan.

Address for reprints: James L. Vacek, MD, 3901 Rainbow Blvd, MS 4023

Kansas City, KS 66160 (E-mail: jlvacek@mac.md).

J Thorac Cardiovasc Surg 2007;133:572-3

$0022-5223 / \$ 32.00$

Copyright @ 2007 by The American Association for Thoracic Surgery

doi:10.1016/j.jtcvs.2006.10.025
Follow-up echocardiography indicated a significant decrease in estimated right ventricular and pulmonary artery systolic pressure (60-65 mm Hg). Severe mitral stenosis was again evident, along with significant tricuspid valve regurgitation. His pressure halftime was $238 \mathrm{~ms}$, with a peak gradient of $49 \mathrm{~mm} \mathrm{Hg}$ and an estimated mitral valve area of $0.9 \mathrm{~cm}^{2}$. The posterior leaflet was tethered to the free wall of the left ventricle, and the anterior leaflet was tethered to the tips of the papillary muscles, with essentially no chordae tendineae. The leaflets were normal in appearance. The valve orifice was fixed and severely restrictive.

At the time of the operation, the echocardiographic diagnoses were confirmed. The patient underwent a complex mitral valve repair within weeks of his initial presentation by using the following techniques: (1) transection of trapped chordae tendineae to both the anterior and posterior mitral valve leaflets from both papillary muscles, leaving normal-sized and normal-appearing pliable leaflets without any chordae tendineae; (2) mitral valve ring annuloplasty with a no. 26 Cosgrove annuloplasty ring; and (3) placement of multiple neochordae (5-0 Gore-Tex polytetrafluroethylene sutures; W.L. Gore and Associates, Flagstaff, Ariz), 2 to the anterior leaflet and 3 to the posterior leaflet. Neochordae were used because of the congenital absence of functional chordae.

After the procedure, the patient had a dramatic reduction in tricuspid regurgitation. He was able to resume activities normal for an adolescent boy without shortness of breath. A postoperative echocardiogram was obtained. The echocardiogram demonstrated no residual mitral stenosis and a trivial jet of mitral valve regurgitation. The Doppler estimated right ventricular systolic pressure was $49 \mathrm{~mm} \mathrm{Hg}$. Moderate tricuspid valve regurgitation was noted. Mitral valve flow on Doppler scanning was $178 \mathrm{~cm} / \mathrm{s}$. Our patient continued to be asymptomatic at 1-year follow-up. 